\title{
Sketch based Shopping
}

\author{
Hraddha. R. Kuchekar ${ }^{1}$, Amruta. L. Satnur ${ }^{1}$, Rutuja. P. Baheti ${ }^{1}$, Prachi. L. Kamble ${ }^{1}$ \\ Student, Computer Department, BVCOEW, Pune, India ${ }^{1}$
}

\begin{abstract}
Content based Image Retrieval is the rising research areas in digital image processing. Sketch Based Image Retrieval is an extension of Content Based Image Retrieval, in which rather than providing a digital image as a query, a hand drawn sketch is used. Sketches are intuitive and descriptive, which offers a more natural way to provide detailed visual clues than pure text. The main objective of this project is to search the similar images from the database where it matches the given input sketch. Database is nothing but large collection of images and those images are extracted and the feature values of these extracted images are saved. The input image is also extracted to get the feature value. This feature value is compared with the values stored in database. If similarity matches, then that image will be displayed as output. Speed up robust feature descriptor is used to make retrieval process fast.
\end{abstract}

Keywords: CBIR, SBIR, Sobel Operator, SURF.

\section{INTRODUCTION}

Image processing is a method to convert an image into digital form and Perform some operations on it in order to get an enhance image or to extract some useful information from it. It involves the manipulation of images to extract information to emphasize or de-emphasize certain aspects of information contained in the image, or to perform statistically or other analysis, to extract non-image information. Thus it is a branch of information technology [10].

\section{CONTENT BASED IMAGE RETERIEVAL}

Content based image retrieval is a retrieval technique which used to retrieve image based on users query input. Content means the features of image such as color, shape, texture etc. or any other described by the image. Content Based Image Retrieval is an automatic process to search relevant images based user query.

CBIR is a technique which uses visual contents to search images from large image databases according to users' interest.

\section{III.SKETCH BASED IMAGE RETRIEVAL}

SBIR is an extension of Content Based Image Retrieval in which rather than providing a digital image as a query, a hand-drawn sketch is used. Sketch is a simple stroke-based drawing, one of its more relevant features is the shape, size, color, stroke orientation etc.

The sketch is drawn by user on the user panel related to the images to be retrieved. It is more feasible than CBIR as to find an image depending upon your expectation can be completed by drawing the figure in your mind but to find an image related to your expectation in order to give it as a query to CBIR system for searching related images is a difficult task as that image may not be available with the user But sketch based image retrieval sorts this problem of having an image for query in hand by providing the user the flexibility to draw the image sketch.

\section{IV.LITERATURE SURVEY}

Ms. Asmita et al, in this system the Edge Histogram Descriptor (EHD) \& Histogram of Oriented Gradients (HOG) feature vector combined together. The edge feature is compared between input image and database image, when matches the feature vector similar image is display on the screen [1].

Shilpa Pawar et al, in this paper, handle the informational gap between a sketch and a painted image.in these paper the HOG and K-mean used together to overcome the drawback in individual that is $\mathrm{k}$-mean is not used for large database but HOG used for large database. These retrieve the image from the database more efficiently than EHD \& HOG [2].

Prasad Mahale et al, SURF standard deviation algorithm is used for matching input image with database distance ratio which is used to determine the matching point the probability of image matching is displayed as output in the form of graph [3].

B. Sz ant o et al, in this paper an implementation is done by using MATLAB. The In these paper Microsoft Research Cambridge Object Recognition Image Database, Wang, Flickr 160 database is used. Using the SIFT-based multilevel solution the search result list is resend [4].

Jyoti Jain et al, In these paper is improving the quality of searching image, in this technique EHD and HOG method work parallel and normalized result is displayed [5].

Meena $\mathrm{M}$ et al, Slope magnitude method is used along with the gradient operators (Sobel, Prewitt, Robert and Canny) to extract the shape features in form of connected 
boundaries. The efficiency of the system is evaluated by the validation measures such as Global Consistency Error (GCE) and Peak Signal to Noise Ratio (PSNR) and PSNR applied for different edge detection operators. The slope magnitude method can be well employed for MRI image analysis [6].

\section{PROPOSED SYSTEM}

In the proposed system for improving the efficiency of an image retrieval system, we are using SURF descriptor. SURF is nothing but speed up robust feature descriptor. It is fast as compared to scale In-variant Feature descriptor.

For gray images, there are many edge detection algorithms such as Robert operator, Sobel operator, Kirsch operator and so on.

Sobel edge detection is gray image processing method. This paper presents a design of a Sobel edge detection algorithm to find edge pixels in gray scale image. In gray scale image each pixel is represented by 8 bit; hence, gray level values vary from 0 to 255 where 0 value stands for black color and 255 values stands for white color. Sobel operator is a widely used method to compute gradient because of its easy format and noise insensitivity character. In gray image, the row and column Sobel operator are defined separately as follows:

$\mathrm{Gy}=$

$\begin{array}{cccccc}\mathrm{y}= & 0 & 1 & \mathrm{Gx}= \\ -2 & 0 & 2 & 1 & 2 & 1 \\ -1 & 0 & 1 & 0 & 0 & 0 \\ & & -1 & -2 & -1\end{array}$

After reducing weight of central pixel,we get $\mathrm{Gy}=$ $\mathrm{Gx}=$

$\begin{array}{cccccc}-1 & 0 & 1 & 1 & \sqrt{2} & 1 \\ -\sqrt{2} & 0 & \sqrt{2} & 0 & 0 & 0 \\ -1 & 0 & 1 & -1 & -\sqrt{2} & -1\end{array}$

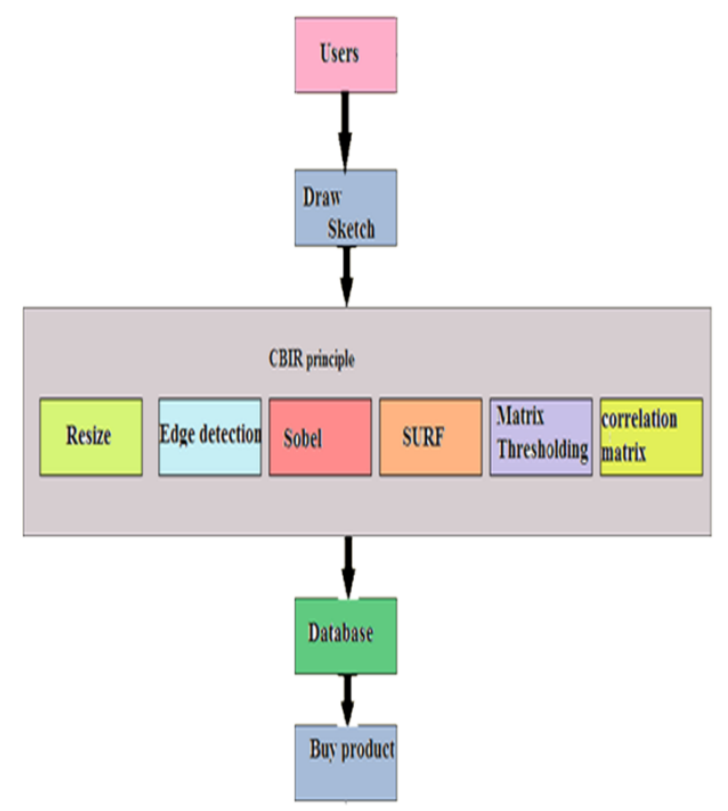

Figure 01: Proposed System Architecture
In proposed system sketch query is nothing but sketch as an input.it is first module of our proposed system. Sketch is a simple stroke-based drawing, one of its more relevant features is the shape, size, color, stroke orientation etc. we are using shape, texture, color feature for extraction. This module is called Feature Extraction Module. In Market there are various Standard database [3] are available Example-flicker 160, Wang, Microsoft research Cambridge object image database, object databank database, sketch images etc.in image database there are number of images are stored to retrieve. For Similarity measure Various Distance Metrics and Similarity Functions are used Euclidean Distance, Manhattan, Chebyshew, Cosine, Hamming etc.

Euclidean Distance: It is well Known Distance Metrics. It used to describe distance between two points or crow flies. It is smaller then, Manhattan distance. Euclidean Distance take shortest path between two points .It used to calculate the distance between query sketch and extracted image. After relevant image will be display. Here, user can select any image for shopping process. After successful shopping user get expected product through this image retrieval proposed system.

1. User - end user

2. Draw sketch- In sketch panel user can draw the free hand sketch and which will be taken as query.

3. CBIR (Content Based Image Retrieval) methods

I. Resize- It will convert drawn sketch image size similar to database image size.

II. Edge detection - It is used for detecting the boundary of the sketches

III.Sobel - Sobel filter detects horizontal and vertical edges separately on a scaled image.

IV.SURF (speed up Robust Feature)- It's approach

is to describes a key point detector and descriptor.

V. Matrix thresholding- Mark pixel point write in matrix format rows and column.

VI. Correlation Matrix-Match the matrix of matrix-thresholding of input image to the matrix of databases images matrix.

4. Database-It consist number of images.

5. Buy product- After matching the given sketch with database it will show the result of online shopping.

\section{VI.ALGORITHM}

I. A.SOBEL EDGE DETECTION.

Pseudo-codes for Sobel edge detection method Input: A Sample Image

Output: Detected Edges

1. Accept the input image

2. Apply mask Gx, Gy to the input image

3. Apply Sobel edge detection algorithm and the gradient.

4. Masks manipulation of Gx, Gy separately on the input image

5. Result to combined to find the absolute magnitude of the gradient

6. $|\mathrm{G}|=\sqrt{\mathrm{a}^{2}+\mathrm{b}^{2}}$ 
7. Where $a=G x, b=G y$

8. the absolute magnitude is the output edges.[9]

\section{PRECISION AND RECALL}

To assess the retrieval effectiveness, we have used the precision and recall as statistical comparison parameters for our proposed technique of CBIR. The standard definitions of these two measures are given by following equations [6]:

Where the Precision provides information related to the effectiveness of the system.

Precision $=$ Sim $/$ Disp

Sim $=$ No. of images displayed with similar shape.

Disp $=$ No. of images displayed.

Where the Recall provides information regarding exact accuracy of the system.

Recall $=$ Sim $/$ Sim_All

Sim $=$ No. of images displayed with similar shape.

Sim_All $=$ No. of images with similar shape in whole database [6]

\section{CONCLUSION}

In the proposed system we are implementing a new searching technique and improving the quality of searching image, in this technique SURF descriptor is used to improve efficiency. To detect edges we are using sobel edge detector operator.

In our proposed system we are extracting color, shape, texture features then by applying some operations on this. Relevant images are retrieved. User can choose one of the expected relevant image from relevant retrieved list to continue shopping.

\section{ACKNOWLEDGMENT}

Our sincere thanks to Head of Department of Computer Science, BVCOEW, Maharashtra, India and my research guide, Department of computer engineering for providing valuable suggestions and guidance in a research work on digital image processing And also sincere thanks for their kind co-operation \& encouragement in carrying out this paper work.

\section{REFERENCES}

[1] Ms. Asmita , Aparna, "Sketch Based Image Retrieval System", International Journal of Advanced Research in Computer Engineering \& Technology (IJARCET) Vol. 3 I(9), 2014

[2] Shilpa Pawar, Sonali Tidke et al, "Survey on Sketch Based Image Retrieval System", International Journal of Emerging Technology and Advanced Engineering (IJETAE), Vol.4, (8), 2014

[3] Prasad Mahale et al, "Enhancing content based image retrieval by using sketches" IEEE ,2014
[4] B. Sz'ant'o, P. Pozsegovics, Z. V'amossy, "Sketch4Match Content-based Image Retrieval System Using Sketches", 9th IEEE, 2011

[5] Jyoti Jain et al , "Improved the Existing Method for Sketch Based Image Retrieval System" , International Journal of Advance Research in Computer Science and Management Studies (IJARCSMS) Volume 1, (3), 2013

[6] Meena M., Priyadharshini B. et al, "Shape Feature Extraction of Brain MRI Using Slope Magnitude Method for Efficient Image Analysis ", International Journal of Innovative Research in Science (IJIRSET), Engineering and Technology Volume 4, Special Issue 11, September 2015.

[7] Neetesh Prajapati, G.S.Prajapti, "Sketch Based Image Retrieval System for the Web" - A Survey International Journal of Computer Science and Information Technologies (IJCSIT), Vol.6 (4), 2015

[8] Geng Xin, Chen Ke, Hu Xiaoguang "An improved Canny edge detection algorithm for color image", 2012 IEEE .

[9] O. R. Vincent, O. R. Vincent "A Descriptive Algorithm for Sobel Image Edge Detection" Proceedings of Informing Science \& IT Education Conference (InSITE) 2009

[10] Madhuri A. Joshi, "Digital Imahe Processing An Algorithminc Approach", PHI learning private limited, New Delhi-110001, ISBN -978-81-203-2971-3,June 2010.

\section{BIOGRAPHIES}

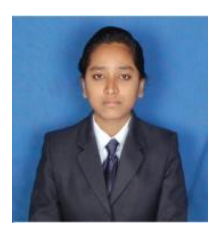

Kuchekar Shraddha Raju B.E. (Computer Engineering), Bharati Vidyapeeth College of Engineering for Womens, Katraj-Dhankwadi, Pune, India Research Area: Image Processing (Edge Detection)

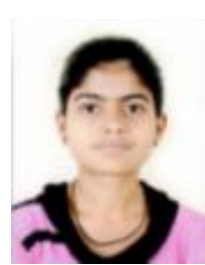

Satnur Amruta Laxmikant B.E. (Computer Engineering), Bharati Vidyapeeth College of Engineering For Womens, Katraj-Dhankwadi, Pune, India Research Area: Image Processing (Edge Detection)

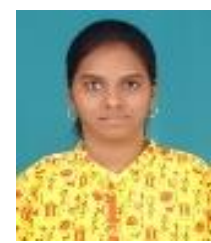

Kamble Prachi Laxman B.E. (Computer Engineering), Bharati Vidyapeeth College of Engineering For Womens, Katraj-Dhankwadi, Pune-43, India

Research Area: Image Processing (Edge Detection) Members of CSIA.

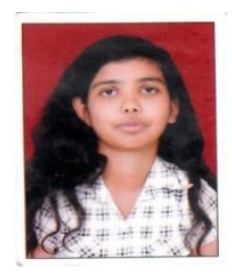

Baheti Rutuja Purushotam B.E. (Computer Engineering), Bharati Vidyapeeth College of Engineering For Womens, Katraj-Dhankwadi, Pune-43, India

Research Area: Image Processing (Edge Detection) Members of 